\title{
Alterstice
}

Revue internationale de la recherche interculturelle

International Journal of Intercultural Research

Revista International de la Investigacion Intercultural

\section{Violence agie ou subie par les jeunes issus de la diversité culturelle : bilan de la recherche québécoise}

\section{Kristel Tardif Grenier, Chantal Lavergne et Janet Sarmiento}

Volume 6, numéro 2, 2016

URI : https://id.erudit.org/iderudit/1040631ar

DOI : https://doi.org/10.7202/1040631ar

Aller au sommaire du numéro

Éditeur(s)

Alterstice

ISSN

1923-919X (numérique)

Découvrir la revue

Citer cet article

Tardif Grenier, K., Lavergne, C. \& Sarmiento, J. (2016). Violence agie ou subie par les jeunes issus de la diversité culturelle : bilan de la recherche québécoise. Alterstice, 6(2), 41-58. https://doi.org/10.7202/1040631ar
Résumé de l'article

Depuis les années 2000, les études sur la violence agie ou subie par les jeunes issus de la diversité culturelle sont de plus en plus nombreuses. Le présent bilan vise à recenser les études québécoises portant sur ce sujet et à faire l'inventaire des caractéristiques de ces études ainsi que de leur objet de recherche. Au total, 44 études portant surtout sur la maltraitance mais également sur la délinquance ont été recensées. Une réflexion sur les forces et les limites de ces études ainsi que sur les pistes prometteuses pour la recherche à venir est présentée en discussion.
Droits d'auteur (C) Kristel Tardif-Grenier, Chantal Lavergne et Janet Sarmiento, 2016
Ce document est protégé par la loi sur le droit d'auteur. L'utilisation des services d'Érudit (y compris la reproduction) est assujettie à sa politique d'utilisation que vous pouvez consulter en ligne. 


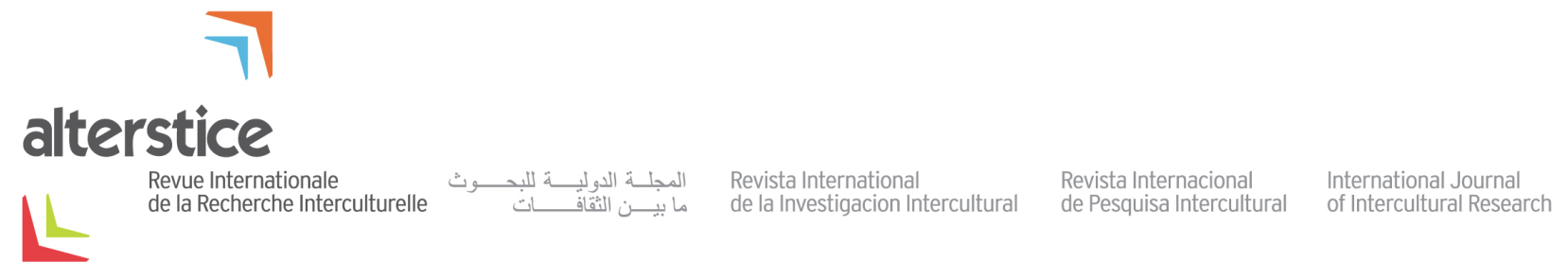

ARTICLE HORS THÈME

\title{
Violence agie ou subie par les jeunes issus de la diversité culturelle : bilan de la recherche québécoise
}

\author{
Kristel Tardif Grenier ${ }^{1}$, Chantal Lavergne ${ }^{2}$ et Janet Sarmiento ${ }^{2}$
}

\section{Résumé}

Depuis les années 2000, les études sur la violence agie ou subie par les jeunes issus de la diversité culturelle sont de plus en plus nombreuses. Le présent bilan vise à recenser les études québécoises portant sur ce sujet et à faire l'inventaire des caractéristiques de ces études ainsi que de leur objet de recherche. Au total, 44 études portant surtout sur la maltraitance mais également sur la délinquance ont été recensées. Une réflexion sur les forces et les limites de ces études ainsi que sur les pistes prometteuses pour la recherche à venir est présentée en discussion.

\section{Rattachement des auteures \\ ${ }^{1}$ Université du Québec en Outaouais, Canada; ${ }^{2}$ Centre de recherche sur les jeunes en difficulté - Centre intégré universitaire de santé et de services sociaux du Centre-Sud-de-l'île-de-Montréal, Canada}

\section{Correspondance}

kristel.tardif-grenier@uqo.ca

\section{Mots clés}

diversité culturelle; violence agie; violence subie; protection de l'enfance; Loi sur le système de justice pénale pour les adolescents; immigration; Autochtones

\section{Pour citer cet article}

Tardif Grenier, K., Lavergne, C. et Sarmiento, J. (2016). Violence agie ou subie par les jeunes issus de la diversité culturelle : bilan de la recherche québécoise. Alterstice, 6(2), 41-58. 


\section{Introduction}

Depuis l'implantation de la mission universitaire en Centre jeunesse au milieu des années 1990, la recherche sur la violence agie et subie par les jeunes a connu un essor considérable au Québec (MSSS, 2005). La violence agie renvoie à la délinquance et aux troubles de comportements alors que la violence subie fait référence à la maltraitance ainsi qu'à la négligence parentale (CIUSS du Centre-Sud-de-l'île-de-Montréal, 2015). Ces deux pôles de la violence sont en lien puisque la violence subie à l'enfance représente un facteur de risque de la délinquance à l'adolescence (Hélie et Laurier, 2012). Or, on constate que la dimension culturelle est de plus en plus souvent prise en compte dans les études portant sur la violence agie ou subie par les jeunes, possiblement en raison du fait que les jeunes issus de la diversité culturelle représentent plus de $20 \%$ de la clientèle recevant des services de protection de l'enfance (Désy, Battaglini et Bastien, 2007). La notion de groupe issu de la diversité culturelle privilégiée dans notre bilan se rapporte aux jeunes issus de l’immigration ou appartenant aux groupes racialisés tels que les Autochtones. Le terme " minorité visible » est aussi fréquemment utilisé pour qualifier la clientèle issue de la diversité culturelle. II s'agit des « personnes, autres que les Autochtones, qui ne sont pas de race blanche ou qui n'ont pas la peau blanche » (Statistique Canada, 2010, p. 1).

L'augmentation de la production scientifique québécoise portant sur la question de la violence agie ou subie par les jeunes issus de la diversité culturelle survient dans un contexte légal exigeant de la part des établissements de santé et de services sociaux de rendre leur offre de services adaptée aux besoins culturels de leur clientèle. En effet, des énoncés de principe sur l'importance de prendre en considération les différences ethniques, culturelles et linguistiques dans l'intervention se retrouvent dans les principales lois régissant la jeunesse en difficulté, soit la Loi sur les services de santé et de services sociaux (LSSSS), la Loi sur la protection de la jeunesse (LPJ) et la Loi sur le système de justice pénale pour les adolescents (LSJPA). Des mesures particulières d'accommodement visant la prestation de services et la détermination de la peine dans le cas des jeunes autochtones existent aussi dans la LPJ et dans la LSJPA. Ainsi, la recherche sur la violence agie et subie par les jeunes issus de la diversité culturelle représente un premier pas vers une potentielle offre de services adaptés aux besoins spécifiques de cette clientèle puisque, pour ce faire, il est d'abord nécessaire d'identifier ses besoins. Cette adaptation représente un défi de taille compte tenu de l'hétérogénéité qui prévaut au sein de la population issue de la diversité culturelle et du fait que chaque culture et les personnes qui en sont issues possèdent des valeurs, des croyances et des pratiques éducatives diversifiées (Morneau, 1999).

Si des bilans des études menées au Royaume-Uni et aux États-Unis portant sur la violence agie ou subie par les jeunes issus de la diversité culturelle sont disponibles (Chand et Thoburn, 2005; Feiring et Zielinski, 2011; Petersen, Joseph et Feit, 2015), il n'en existe aucun à notre connaissance sur les études québécoises. Notre bilan vise donc à contribuer à l'amélioration des connaissances sur la violence agie et subie par les jeunes issus de la diversité culturelle et à décrire les caractéristiques méthodologiques des études menées à ce sujet. Les forces et les lacunes de ces études sont identifiées et des pistes pour la recherche à venir sur ce sujet sont suggérées en guise de conclusion.

\section{Méthodologie}

\section{Repérage et sélection des études}

Une recherche bibliographique a été effectuée à l'aide de plusieurs bases de données, dans le but de repérer les études pertinentes aux fins de cette recension: Dialog, Santécom, Érudit, Sign@l, Atrium et Thèses Canada. Certains sites pertinents tels que ceux du Portail canadien de la recherche sur la protection de l'enfance, de METISS, du SHERPA (recherche, immigration, société) et d'Alterstice ont été consultés, avec les mots-clés suivants : protection de la jeunesse, maltraitance envers les enfants, placement, délinquance, services sociaux aux jeunes en difficulté, prévention, child welfare, jeunes réfugiés, issus de l'immigration et des minorités visibles, enfants et jeunes autochtones.

Des critères de sélection des études à retenir pour ce bilan ont été établis. D’abord, pour faire partie du bilan, les travaux de recherche devaient être basés sur une cueillette de données primaires ou sur une analyse de données 
secondaires de type quantitatif, qualitatif ou documentaire. Les recensions d'écrits ont aussi été incluses dans notre échantillon. Les recherches devaient être produites au Québec ou concerner des populations situées au Québec. Les études sélectionnées pouvaient être publiées en français ou en anglais, sous forme d'articles scientifiques, de rapports de recherche, de publications institutionnelles, de thèses de doctorat et de mémoires de maîtrise. Enfin, la recherche documentaire a couvert les publications parues entre janvier 2000 et octobre 2014.

\section{Paramètres de classement des études}

Cinq caractéristiques ont été retenues pour procéder au classement des études retenues pour notre bilan. Deux analystes ont mené cette démarche de façon indépendante. D'abord, le type de méthode d'analyse des données (qualitative ou quantitative) a été pris en considération. Ensuite, chaque étude recensée a été située sur le continuum d'intervention auprès des jeunes en difficulté, qui comporte quatre étapes : prévention, intervention, réadaptation et réinsertion (Chamberland, 1998; Tremblay, Moisan, Laquerre et Faugeras, 2002). La prévention concerne les actions menées en amont du signalement et visant à éviter l'apparition de problèmes sociaux chez les jeunes et leur famille. L'intervention renvoie à l'action des centres jeunesse relativement à l'évaluation et à l'orientation des situations ainsi qu'à l'application de mesures de protection prenant place dans la communauté ou en milieu familial. La réadaptation fait référence aux interventions menées en contexte d'hébergement ou de placement en Centre jeunesse. La réinsertion traduit l'accompagnement du jeune durant la transition entre I'hébergement et la réinsertion dans son nouveau milieu de vie. Enfin, le niveau de participation au processus de recherche de membres des communautés étudiées a aussi été pris en compte dans le classement des études. Le modèle de Wells, Miranda, Bruce, Alegria et Wallerstein (2004) suggère que les recherches qualifiées de participatives doivent permettre à des représentants de ces communautés de participer à au moins deux des étapes suivantes: l'élaboration de la question de recherche, l'interprétation des données et la diffusion des résultats. Enfin, la composition et le groupe d'âge des populations étudiées ainsi que la région administrative du Québec couverte par l'étude ont aussi été pris en considération dans le classement des études. Dans les cas de divergence sur les caractéristiques des études, des discussions de groupe ont permis d'atteindre un consensus.

\section{Résultats}

\section{Rythme de la production et caractéristiques des études}

Au total, 44 études ont été recensées. La figure 1 illustre la répartition des travaux de recherche recensés selon leur année de parution. On constate que les études sont peu nombreuses jusqu'au milieu de la décennie, mais que leur nombre augmente à partir de 2009.

Figure 1 Répartition du nombre de publications par année

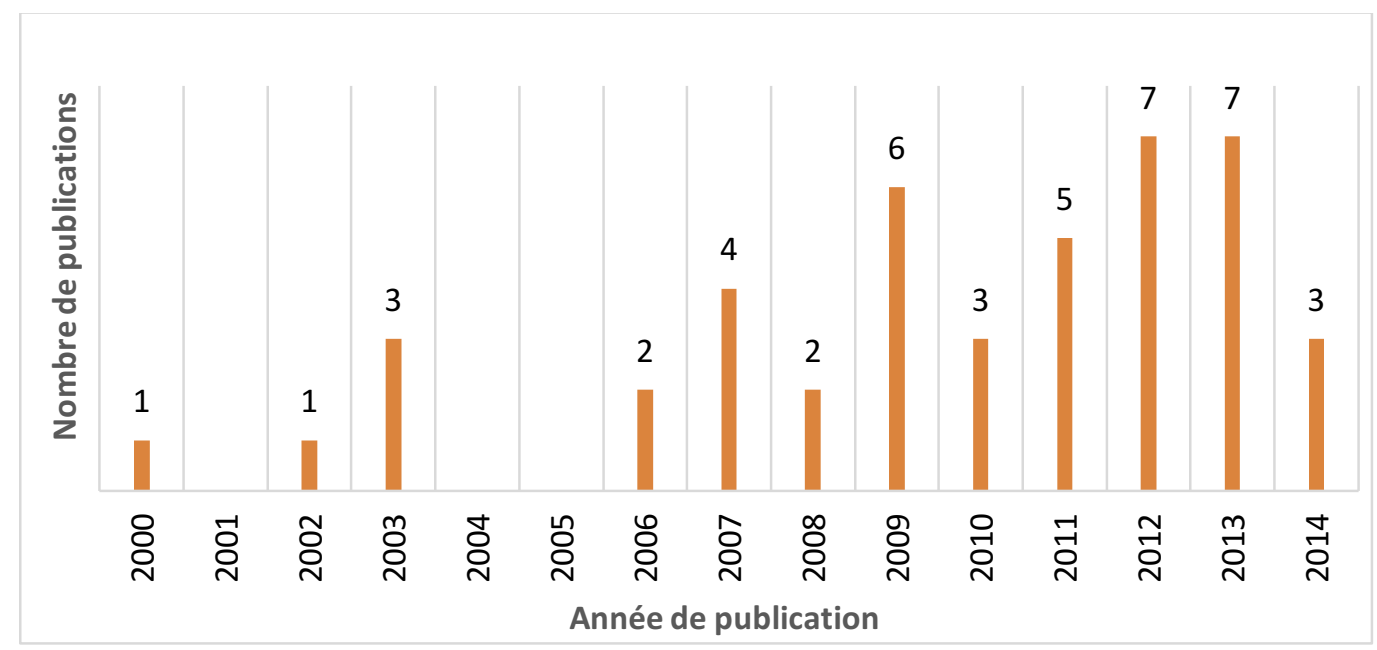


Bien que les études recensées présentent des caractéristiques variées (tableau 1), certaines tendances se dégagent. Tant en ce qui a trait aux études qui portent sur les jeunes autochtones que celles qui s'intéressent aux jeunes issus de l'immigration ou des minorités visibles, les méthodologies qualitative et quantitative sont privilégiées presque également, alors que l'approche mixte l'est nettement moins. On constate aussi un équilibre quant au type d'ouvrage, puisqu'on compte 13 mémoires de maîtrise, 14 articles publiés dans des revues avec comité de lecture et 14 rapports ou publications institutionnelles. On recense également une thèse de doctorat, un chapitre de livre et un livre.

Les études de nature descriptive ou exploratoire dominent et très peu d'études ont opté pour un devis permettant de comparer des jeunes issus de différentes communautés et donc de cerner leurs besoins spécifiques. Les études portent, dans une proportion équivalente, sur les jeunes et leurs familles issus de communautés autochtones et sur ceux issus de l'immigration. Plusieurs études ont cependant été menées auprès de jeunes haïtiens et les études sont moins nombreuses à porter sur les autres communautés présentes au Québec. Relativement peu d'études portent sur un groupe d'âge spécifique, et bon nombre d'entre elles englobent plutôt l'ensemble des jeunes de dixsept ans et moins. Les adolescents et les jeunes adultes font exception, puisque le tiers des études porte spécifiquement sur ce groupe d'âge. Les études couvrent dans une proportion presque similaire Montréal et les autres régions ou l'ensemble du territoire québécois. En ce qui a trait au continuum d'intervention, l'étape de l'intervention se retrouve dans 31 études, suivie de loin par la prévention (7 études) et la réinsertion (5 études). Une seule étude traitait de l'étape de la réadaptation. On constate aussi une disproportion au niveau des objets de recherche des études recensées. En effet, celles-ci sont nettement plus nombreuses à porter sur la violence subie que sur la violence agie. En ce qui a trait au niveau de participation de membres de la communauté au processus de la recherche (Wells et al., 2004), il ressort que très peu d'études sont basées sur des collaborations actives entre les chercheurs et les représentants des populations étudiées, à l'exception d'une recherche-action menée en collaboration avec des membres de la communauté "noire » de Montréal et de quelques études avec des membres de communautés autochtones.

\section{Objets de recherche des études recensées}

En plus de décrire les caractéristiques méthodologiques des études recensées, notre bilan vise à faire état des connaissances qui en sont issues. Pour ce faire, les études ont été classées selon deux axes : la violence subie par les jeunes issus de la diversité culturelle (1) et la violence agie par les jeunes issus de la diversité culturelle (2). Les détails concernant l'échantillon, la méthodologie et les objectifs de chacune des études sont rassemblés dans le tableau 1.

\section{Axe 1 : Violence subie par les jeunes issus de la diversité culturelle}

La surreprésentation des enfants autochtones. L'intervention des services de protection de l'enfance est nécessaire lorsqu'un enfant est victime de violence comme des actes de maltraitance. Or la surreprésentation des enfants autochtones dans le système de protection de l'enfance est un thème exploré dans plusieurs études, tant par le biais de portraits quantitatifs que de points de vue d'acteurs clés. Une étude montre à cet égard que ces enfants sont surreprésentés à toutes les étapes de la prise de décision et que cette surreprésentation augmente à mesure qu'ils progressent dans leur trajectoire de services en protection de l'enfance (Breton, Dufour et Lavergne, 2012). Le fait d'être Autochtone double la probabilité de faire l'objet d'un placement, même lorsqu'on tient compte simultanément d'un ensemble de caractéristiques pouvant contribuer au placement. Dans le même sens, Hélie, Laurier, Pineau-Villeneuve et Royer (2013) suggèrent que le fait d'être d'origine autochtone augmente aussi le risque de récurrence en protection de l'enfance durant les cinq ans suivant la fermeture de la première prise en charge. Des explications de cette surreprésentation sont évoquées par Tourigny, Domond, Trocmé, Sioui et Baril (2007) dans leur étude qui montre que, comparativement aux jeunes non autochtones, les jeunes autochtones signalés ont un profil psychosocial nettement plus lourd. En effet, les enfants autochtones vivent plus souvent dans une famille nombreuse et sont plus enclins à habiter un logement non conforme aux normes et à avoir des parents aux prises avec plus de problèmes d'abus de substances que les parents des enfants non autochtones. 
La surreprésentation des jeunes issus de l'immigration et des minorités visibles. Des études ont porté sur la surreprésentation des jeunes issus de l'immigration ou des minorités visibles. Dufour, Hassan et Lavergne (2012) parviennent ainsi à la conclusion que les enfants " noirs » sont près de deux fois plus enclins que les autres à faire l'objet d'un signalement, d'être considérés en besoin de protection ou d'être placés. À l'inverse, les enfants issus des autres minorités visibles sont les moins susceptibles de recevoir des services de protection et leur sousreprésentation tend à croître à chaque étape de la trajectoire. La surreprésentation des enfants " noirs » au sein des services de protection de l'enfance serait attribuable à divers facteurs tels que la pauvreté et la discrimination raciale. Garland (2009) suggère pour sa part que les jeunes « noirs » et les jeunes asiatiques sont surreprésentés parmi les adolescents ayant un signalement lié à un abus physique. Pourtant, Lavergne, Dufour, Sarmiento et Descôteaux (2009) concluent que les caractéristiques des enfants et des familles appartenant à un groupe de minorité visible paraissent plus enviables que celles des enfants qui ne sont pas issus de ces groupes. En effet, ces enfants requièrent moins de services en protection et font moins souvent l'objet d'un placement à l'étape de l'application des mesures. D’autres chercheurs ont réfléchi aux facteurs explicatifs de cette surreprésentation. Belony (2007) suggère par exemple que la surreprésentation des enfants "noirs" au sein des services de protection de l'enfance est le produit d'un cumul de facteurs de risques (monoparentalité, faible revenu et faible scolarité parentale) qui rendrait ces enfants plus susceptibles d'être pris en charge. Lavergne, Dufour et Couture (2013) ont, eux aussi, identifié des facteurs explicatifs à la surreprésentation des enfants " noirs ", en particulier les difficultés liées au processus migratoire et à l'insertion dans la société d'accueil.

Les causes de la violence subie par les jeunes. Au-delà du phénomène de la surreprésentation, des chercheurs ont tenté de cerner les causes de la violence subie par les jeunes issus de la diversité culturelle. Il s'agit d'une question délicate dans la mesure où il faut prendre garde à ne pas "culturaliser » les formes de violences subies par ces jeunes, c'est-à-dire désigner la culture comme facteur explicatif des conduites inappropriées. C'est l'une des mises en garde qui ressort de l'étude d'Hassan et Rousseau (2009), qui fait état d'une grande diversité d'opinions à l'égard de l'acceptabilité des châtiments corporels au sein de deux groupes d'immigrants originaires du Maghreb et d'Amérique Latine. Par ailleurs, les facteurs en cause dans les agressions sexuelles, une forme de violence subie, ont attiré l'attention de chercheurs s'intéressant aux jeunes autochtones. Morin et Lafortune (2008) ont dégagé des caractéristiques attribuables au contexte nordique et pouvant contribuer à la prévalence élevée des agressions sexuelles observée au sein de certaines communautés inuites telles que l'importance du patriarcat dans la vie ancestrale, la consommation d'alcool, l'isolement géographique et l'étroitesse des rapports interpersonnels. Cette étude comporte une dimension participative, puisque des leaders de la communauté y ont contribué et que les intervenants participant à l'étude étaient issus de trois villages nordiques. Muckle (2011) a pour sa part documenté des facteurs de résilience chez des jeunes autochtones ayant subi ce type d'agression sexuelle : l'estime de soi et la capacité à rechercher de l'aide dans la communauté sont associées à une résilience accrue chez ces adolescents.

Les autres formes de violences subies. Outre la maltraitance, il existe d'autres formes de violences subies par les jeunes. À cet égard, une étude permet de mettre en lumière le fait que la discrimination représente l'une des violences subies par les jeunes issus des minorités visibles, en particulier les jeunes "noirs ". L'étude comparative de Bernard et McAll (2009) suggère l'existence de profilage racial chez les policiers à l'égard des jeunes « noirs ». En effet, parmi les 1518 jeunes ayant été arrêtés en 2001 sur l'île de Montréal, près du quart ont été identifiés par les policiers comme "noirs", un pourcentage deux fois supérieur à la proportion de ces jeunes dans la population. Les violences basées sur l'honneur telles que les crimes d’honneur, les mariages forcés et les mutilations génitales représentent d'autres formes de violence subie qui touche certaines communautés. Le Conseil du statut de la femme (2013) a cherché à faire la lumière sur les cas de violences basées sur l'honneur ayant fait l'objet d'une judiciarisation au Canada. L'un des constats formulés est qu'il y a eu une augmentation marquée de ces cas à partir de 2001. Il est possible de dégager quatre principaux motifs aux violences basées sur l'honneur visant principalement des femmes en âge de procréer : la volonté d'autonomie de la victime face au contrôle parental, le désir de choisir son propre conjoint, le fait de vouloir divorcer ou celui d'être soupçonnée d'infidélité. Ces situations sont d'autant plus préoccupantes que les jeunes immigrants exposés à la violence vivent davantage de stress que leurs pairs non immigrants se trouvant en situation comparable. En effet, Jimenez et al. (2003) ont montré que les enfants immigrants exposés à la violence conjugale voient leur santé mentale davantage affectée en raison d'un cumul de facteurs de stress attribuables tant à la violence subie qu'à l'immigration. 
Les particularités propres à l'intervention en contexte de diversité culturelle. Si les jeunes issus de la diversité culturelle sont surreprésentés au sein des services de protection de l'enfance, qu'en est-il de leurs besoins spécifiques et des particularités de l'intervention en contexte de diversité culturelle ? Hassan et Rousseau (2007) rapportent que les intervenants en protection de l'enfance constatent que leurs interventions suscitent des sentiments de méfiance, de peur et de honte chez les familles immigrantes auprès desquelles ils interviennent. Dans la même veine, Désy, Battaglini et Bastien (2007) se sont penchés sur la nature et l'intensité de l'intervention en protection de l'enfance auprès de jeunes issus de l'immigration et de leur famille. Certaines caractéristiques telles qu'une langue de communication autre que le français ou l'anglais ainsi qu'une méconnaissance du fonctionnement des institutions québécoises rend l'intervention auprès de cette clientèle plus complexe et nécessite un temps d'intervention jusqu'à deux fois plus long. Dans le même ordre d'idées, Leblanc (2003) fait ressortir le fossé culturel qui peut exister entre les intervenants en protection de l'enfance et les communautés autochtones. Ces études renforcent la pertinence du développement de pratiques spécifiques à la protection des jeunes issus de la diversité culturelle et certaines études ont visé à décrire et analyser ces pratiques.

L'étude de Bousquet St-Laurent, Dufour et Lavergne (2014) recense 29 pratiques associées à la diversité ethnoculturelle en protection de l'enfance. Ce bilan met en évidence le fait que le soutien aux familles et l'adaptation des services et protocoles de protection sont les pratiques les plus courantes, et qu'elles impliquent souvent de solliciter le point de vue ou la collaboration de membres de la communauté concernée. Aucune de ces pratiques ne respecte cependant les critères qui permettraient de la qualifier d'efficace ou de probablement efficace. Couture et Dufour (2013) ont analysé les pratiques des intervenants en protection visant la collaboration avec les familles de minorités visibles. Selon les intervenants rencontrés, la lecture des besoins et la description des caractéristiques familiales est nécessairement teintée de la culture et des valeurs personnelles de l'intervenant ainsi que du cadre de référence institutionnel, ce qui peut donner lieu à une résistance à la collaboration chez les parents. Il est donc important de parvenir à une lecture commune de la situation entre l'intervenant et les parents. Afin d'améliorer les relations entre les intervenants en protection de l'enfance et les familles issues de l'immigration, le programme "Vivre la diversité » a été mis en place. La mise en œuvre de ce programme ainsi que ses effets ont été évalués par Giroux (2012). Des changements significatifs ont été observés au sein du groupe d'intervenants ayant bénéficié du programme, en partiuclier l'augmentation des connaissances générales relatives aux relations interculturelles, mais aucun changement n'a été observé sur le plan des attitudes et des habiletés professionnelles.

D'autres pratiques sont destinées plus spécifiquement à la clientèle autochtone. C'est notamment le cas de groupes de soutien pour jeunes autochtones en transition vers la vie adulte pris en charge par un Centre jeunesse. L'implantation et les effets de ce programme d'intervention ont été évalués dans différentes régions du Québec par Goyette et al. (Goyette, Grenier, Pontbriand et al., 2009; Goyette, Grenier, Mann-Feder et al., 2010; Goyette, Mann-Feder, Turcotte et al., 2012) ainsi que par Grenier, Goyette, Turcotte, Mann-Feder et Turcotte (2013). Ces auteurs mettent en lumière le fait que le groupe représente pour ces jeunes une condition facilitante dans le processus de construction identitaire et constitue un outil de médiation permettant le rétablissement de liens sociaux fragilisés. McGinn et Damasse (2014) ont analysé la documentation sur l'efficacité de trois programmes visant à développer les habiletés parentales des Autochtones en contexte de négligence et ont conclu que ces mesures affichent une efficacité mitigée. Robitaille (2011) a traité de l'influence de la culture autochtone sur la prise de décisions cliniques en protection de l'enfance et montre que cette culture peut être perçue par les intervenants comme un élément facilitant l'intervention parce qu'il représente un outil de mobilisation. Elle peut toutefois aussi être appréhendée par les intervenants comme un défi, voire un obstacle, ou un indice de vulnérabilité. Sur le plan organisationnel, Sigouin (2006) a examiné quatre aménagements institutionnels ayant pour objectif de donner plus de contrôle aux Autochtones en matière de protection de l'enfance. Le système permettant une reconnaissance du droit à l'autonomie gouvernementale, c'est-à-dire qui octroie des pouvoirs législatifs aux Autochtones, est le plus approprié pour concilier les droits individuels et les droits collectifs de ces derniers. Enfin, Sinha et Kozlowski (2013) ont procédé à une analyse documentaire afin de brosser un portrait de la structure des services de protection de l'enfance spécifiques aux enfants autochtones du Canada. II existe entre les agences autochtones de protection de l'enfance une grande variation dans les modèles d'organisation des services et de financement. À la différence d'autres provinces, les lois et les modèles québécois d'organisation de services 
n'incluent pas l'obligation d'informer les autorités autochtones du placement d'un enfant autochtone ni celle d'impliquer des représentants autochtones dans la gestion de cas en protection de l'enfance.

L'intervention en protection de l'enfance menée auprès de familles issues de la diversité culturelle est parfois complexe, notamment en raison d'une divergence de points de vue entre familles et intervenants (Désy, Battaglini, et Bastien, 2007). Pour cette raison, des chercheurs se sont intéressés au point de vue de parents et de jeunes relativement aux services de protection. Ainsi, Labonté (2010) rapporte que l'intervention en protection de l'enfance est parfois perçue par les parents réfugiés comme une remise en question de leur modèle familial par l'imposition d'une éducation nord-américaine. Gagnon-Dion (2014) s'est penchée sur le point de vue des jeunes autochtones quant à leur propre parcours de prise en charge et a identifié trois profils : les "résistants », qui ont de la difficulté à accepter leur prise en charge, les "résilients", qui vivent positivement leur placement, et les "résignés ", qui semblent y être indifférents. Certains membres d'une communauté autochtone perçoivent qu'il serait souhaitable que les familles soient davantage engagées dans les démarches entreprises par les services de protection de l'enfance et que la recherche de solutions devrait en premier lieu se faire au sein de la famille proche et élargie (Mitchell, 2011).

Un autre sujet ayant retenu l'attention des chercheurs est celui de la fixation de délais maximaux de placement, qui représente l'une des plus importantes modifications apportées à la LPJ en 2007. Ce changement à la loi vise à faciliter l'adoption des enfants en bas âge et fait en sorte qu'un parent peut définitivement perdre ses droits parentaux. Cette mesure a toutefois fait l'objet de vives critiques de la part de représentants autochtones, qui craignent qu'elle ne favorise l'exil des enfants autochtones à l'extérieur des communautés. C'est dans l'optique de mieux cerner la nature de ces résistances qu'Abdel Ghaly (2013) s'est penchée sur les représentations de huit femmes autochtones relativement aux délais de placement maximaux. La plupart des femmes rencontrées s'y opposent et formulent des inquiétudes quant à l'incidence de ces délais sur le tissu social des communautés ainsi que sur la préservation de l'identité culturelle des enfants. Dans le même ordre d'idées, deux études d'Hélie et al. (Hélie, Turcotte, Royer et Lamonde 2011; Hélie, Turcotte, Trocmé et Tourigny, 2012) ont permis de documenter une légère augmentation des taux de placement chez les enfants autochtones suite à ces changements législatifs alors que la tendance inverse est observée pour les enfants non autochtones. On constate cependant une baisse des sorties non permanentes chez les enfants autochtones, c'est-à-dire des sorties du réseau des services de santé et sociaux suivies d'un nouvel épisode de placement. Sur une note plus positive, Goyette, Pontbriand et Turcotte (2011) rapportent que les modifications apportées à la LPJ qui apparaissent comme les plus adaptées au contexte autochtone sont celles qui limitent le processus de judiciarisation et favorisent le recours à la famille élargie et à la communauté.

Outre le thème de la violence subie, celui de la violence agie a également attiré l'attention des chercheurs.

\section{Axe 2 : Violence agie par les jeunes issus de la diversité culturelle}

Comme nous l'avons évoqué plus tôt, certaines communautés sont surreprésentées dans le système pénal juvénile, notamment en raison de la discrimination dont elles font l'objet (Bernard et McAll, 2009). Parmi les violences susceptibles d'être agies par ces jeunes, on retrouve l'engagement dans des conduites délinquantes.

Les jeunes issus de la diversité culturelle et la délinquance. Des études se sont attardées aux facteurs de risque de la délinquance chez les jeunes issus de la diversité culturelle. Par exemple, Grobe (2000) s'est penchée sur le type de relation d'attachement parent-adolescent autochtone comme prédicteur des problèmes de comportement. L'auteure parvient au constat surprenant que les jeunes ayant développé une relation d'attachement optimale avec leurs parents sont plus à risque de délinquance que les jeunes ayant des relations d'attachement moins optimales. Ces résultats suggèrent que les théories de l'attachement ne sont possiblement pas applicables à toutes les cultures. Desruisseaux, St-Pierre, Tougas et de la Sablonnière (2002) ont élaboré un modèle explicatif pour rendre compte de l'adoption de conduites déviantes par les jeunes Haïtiens. Plus ces jeunes perçoivent qu'ils subissent un préjudice attribuable à leur origine ethnique, plus ils sont enclins à fréquenter des amis délinquants et à adopter des comportements déviants. Cette étude fait écho à celle de Tichit et Jaccoud (2009), qui rapporte que des jeunes «noirs " perçoivent que les raisons pour lesquelles des jeunes de leur communauté s'impliquent dans 
des gangs de rue sont la discrimination perçue, le manque d'éducation et d'amour, l'influence du groupe, le plaisir de commettre des actes violents ainsi que la quête du pouvoir et de l'argent. Bergheul (2012) a mené une étude comparative afin de cerner les différences sexuelles et culturelles quant au vécu des adolescents délinquants vivant au Québec et en Algérie. II en ressort que les délinquantes algériennes se distinguent de leurs homologues québécoises par une plus grande tendance aux actes enfreignant les règles sociales et conduisant au rejet de leur communauté, ce qui aurait pour effet d'affaiblir leur réseau social et d'aggraver leur délinquance.

Les gangs de rue. Une des manifestations de la violence agie ayant particulièrement attiré l'attention est celle de l'affiliation aux gangs de rue. Plusieurs chercheurs ont tenté d'en cerner les mécanismes, notamment en recueillant les points de vue de jeunes impliqués au sein de ces groupes marginalisés. Perreault et Bibeau (2003) avancent que pour les jeunes québécois afro-antillais marginalisés, l'appartenance à un gang pallie un manque de lien social en leur permettant de sortir de leur isolement. Ces jeunes nomment que le gang représente pour eux un groupe d'amis qui partagent les mêmes problèmes et qui fournit des occasions de faire de l'argent. De Laco (2006) s'est intéressé au rôle de l'identité culturelle dans l'expérience de jeunes issus de l'immigration à l'égard de leur participation à un gang. Il en ressort que la culture jouerait un rôle primordial dans l'appartenance au gang pour les jeunes latinos, qui y trouvent un moyen de préserver leur identité culturelle et de s'opposer au racisme dont ils perçoivent faire l'objet. Portant aussi sur les jeunes latinos, l'étude de Tremblay (2008) met en évidence certains facteurs explicatifs de l'adhésion de ces jeunes à un gang, qui seraient nombreux à avoir connu la séparation brutale d'avec un être proche et à avoir un membre de leur famille affilié à un gang. Une étude ayant porté sur des parents dont le fils fréquente des gangs de rue a permis de cibler certains facteurs familiaux expliquant cette problématique (Brisebois, 2007). Ces parents rapportent avoir vécu difficilement la confrontation avec les valeurs de la culture d'accueil et avoir adopté des pratiques parentales extrêmes, soit très permissives, soit très autoritaires. Au-delà des facteurs de risques, cette étude permet de retracer le parcours migratoire de ces parents.

Toutefois, les programmes d'intervention visant la réadaptation des jeunes contrevenants issus de la diversité culturelle ont peu attiré l'attention des chercheurs. Une étude a porté sur un programme de réadaptation en hébergement hors territoire pour jeunes inuit (Fraser, Vachon, Arauz, Rousseau et Kirmayer, 2012). Réalisée en collaboration avec des partenaires autochtones, cette étude a permis d'identifier plusieurs éléments facilitant la réintégration de ces jeunes dans la communauté, par exemple le retour à l'école et l'obtention d'un emploi.

\section{Conclusion}

En somme, ce bilan des connaissances sur la violence agie et subie par les jeunes issus de la diversité culturelle témoigne de l'hétérogénéité qui caractérise ce domaine de recherche. On constate que la recherche québécoise portant sur la violence agie ou subie par les jeunes issus de la diversité culturelle a connu un essor important au cours des dix dernières années. Cette tendance ne reflète cependant pas nécessairement un intérêt plus marqué à l'endroit de la clientèle issue de la diversité culturelle, mais plutôt l'expansion des efforts de recherche sur les jeunes en général. Malgré des forces indéniables, comme la diversité des objets abordés et l'équilibre sur le plan des approches méthodologiques privilégiées, plusieurs lacunes ont été constatées dans les études recensées.

\section{Limites méthodologiques des études recensées}

La majorité des études produites appartient à la description et à l'exploration. Les rares études ayant adopté une perspective comparative ont procédé à des regroupements de populations hétérogènes, par exemple les enfants "noirs", qui regroupent des enfants issus des communautés antillaise et africaine et qui présentent des profils différents, ce qui peut affecter la validité des conclusions tirées de ces comparaisons. On dispose également d'un faible nombre d'études longitudinales ou d'évaluations de programme, qui s'avèrent pourtant indispensables pour l'évaluation des efforts d'adaptation des services auprès de ces clientèles. Le manque de recherches évaluatives portant sur les effets des programmes auprès des jeunes issus de la diversité culturelle fait en sorte qu'on ne sait pas si les programmes mis en place fonctionnent auprès de ces populations, ni s'ils sont adaptés à leurs besoins, et cela est d'autant plus vrai pour certains secteurs d'intervention comme la réadaptation qui ont peu retenu l'attention de la recherche. Enfin, très peu d'études font appel à une démarche participative permettant l'implication active de membres des communautés visées dans le processus de recherche. Une telle participation serait pourtant souhaitable, puisqu'elle permettrait notamment d'enrichir l'interprétation des résultats de ces 
études. Ce bilan permet néanmoins de tirer certaines conclusions quant aux besoins et aux pratiques probantes relativement aux jeunes issus de la diversité culturelle, et ce, tant en ce qui a trait à la violence subie qu'à la violence agie.

\section{Violence subie par les jeunes issus de la diversité culturelle : besoins et pratiques probantes}

Les études ayant traité de la violence subie montrent que les jeunes issus de la diversité culturelle sont surreprésentés dans le système de protection de l'enfance et qu'ils présentent souvent un profil désavantagé, notamment en ce qui a trait au statut socioéconomique. Ces caractéristiques combinées à un manque de formation chez les intervenants et à la présence de malentendus inhérents à une divergence sur le plan des valeurs pourrait expliquer cette surreprésentation. Les difficultés associées au processus migratoire et d'insertion dans la société d'accueil peuvent contribuer à rendre les familles plus vulnérables au stress ainsi qu'à une série d'autres problèmes psychosociaux susceptibles de nuire aux relations parents-enfants, d'affecter ou même de compromettre le développement des enfants (Jimenez et al., 2003). Malheureusement, on ne compte que trop peu d'études portant sur les défis et les obstacles à l'intégration des jeunes et de leurs parents issus de l'immigration qui reçoivent des services en protection de l'enfance, ce qui fait que certaines questions demeurent. Comment des facteurs de vulnérabilité comme la pauvreté, la déqualification professionnelle, les problèmes en lien avec la maîtrise d'une des langues officielles, l'incertitude liée au statut vis-à-vis de l'immigration, les chocs culturels, les conflits intergénérationnels et l'éloignement de la famille élargie affectent-ils la capacité des parents immigrants à répondre aux besoins de leurs enfants? Quelles sont les forces de ces familles et les opportunités qui s'offrent à elles dans leur pays d'accueil ? Comment ces parents s'adaptent-ils aux normes et croyances du pays d'accueil en matière d'éducation des enfants et de pratiques disciplinaires?

Les autres formes recensées de violence subie par les jeunes issus de la diversité culturelle sont l'agression sexuelle, la discrimination et les violences basées sur l’honneur. Les études évoquent des pistes d'explication systémiques, individuelles et familiales, illustrant ainsi la grande complexité de ces problématiques. De surcroît, bien que des études aient mis en lumière l'effort supplémentaire en temps et en ressources nécessaire à l'intervention en contexte de diversité culturelle, il est malheureusement impossible d'identifier les pratiques probantes en matière de violence subie par les jeunes. En effet, aucune des pratiques recensées ne rencontre les critères minimaux d'efficacité avérée ou probable (Saunders, Berliner et Hanson, 2004), tels que le recours à un groupe contrôle et un suivi longitudinal relativement au maintien des acquis. Dans une optique de prévention, il est essentiel de s'intéresser aux pratiques fructueuses visant à aider les familles issues de la diversité culturelle à prendre soin des enfants et à empêcher que ces derniers ne soient pris en charge par les services de protection de l'enfance. II serait toutefois erroné de se centrer uniquement sur les facteurs de risque présents dans cette population. Malheureusement, très peu d'études ont traité du recours à l'identité culturelle en intervention ou aux aménagements pouvant être apportés dans les services pour tenir compte de la culture du jeune et de sa famille. II serait notamment pertinent d'envisager le recours à la famille élargie ainsi qu'au pairage ethnoculturel des enfants placés. Nous manquons également d'information sur la collaboration entre les établissements de services sociaux et les organismes ethnoculturels, alors qu'il s'agit d'un moyen encouragé par le législateur pour améliorer les résultats des interventions.

\section{Violence agie par les jeunes issus de la diversité culturelle : besoins et pratiques probantes}

Notre bilan permet de montrer que le thème de la violence agie a été nettement moins exploré que celui de la violence subie. En raison d'une pauvreté économique plus importante et du profilage dont ils font l'objet, les jeunes autochtones et ceux issus de l'immigration sont particulièrement susceptibles de vivre des difficultés sociales, comme des problèmes de délinquance et des démêlés avec la justice pénale (Kroes, 2008). Plusieurs facteurs de risque de délinquance chez les jeunes issus de la diversité culturelle ont été documentés, tels que le fait d'avoir une relation d'attachement sécure, de percevoir une discrimination envers soi et d'être isolé de sa communauté. Des études ont porté sur le phénomène des gangs de rue et permettent de mettre en lumière que ce type de groupe criminalisé permet de répondre à certains besoins affectifs des jeunes qui y adhèrent, en particulier le besoin d'appartenance et d'identification. Il est encore une fois impossible d'identifier les pratiques probantes pour prévenir et traiter les problématiques de violence agie chez les jeunes issus de la diversité culturelle, 
puisqu'une seule étude s'est attardée à l'évaluation d'un programme (Fraser, Vachon, Arauz, Rousseau et Kirmayer, 2012).

Il y a donc matière à poursuivre les efforts de recherche en ce qui concerne la violence agie par les jeunes issus de la diversité culturelle. Dans un premier temps, il serait important de se doter d'un portrait plus précis de ces jeunes lorsqu'ils reçoivent des services en Centre jeunesse en vertu de la LSJPA. On dispose encore de très peu d'information sur la présence, les caractéristiques et les besoins de ces jeunes dans le système québécois de justice pénale pour adolescents. Des études portent sur le parcours de jeunes issus de l'immigration et appartenant aux gangs de rue, mais très peu de chercheurs se sont intéressés à l'implication de ces jeunes dans les autres formes de délinquance et aux voies multiples menant à l'engagement dans la délinquance chez les adolescents autochtones. La situation de ces derniers devrait recevoir une attention particulière à cet égard. Des données permettent en effet de constater l'existence d'une surreprésentation des adolescents autochtones à l'étape de la mise sous garde dans certaines provinces et territoires du Canada, mais pas encore au Québec (Latimer et Foss, 2004).

Somme toute, la croissance démographique au sein des communautés autochtones ainsi que l'arrivée massive de familles immigrantes au Québec renforcent la pertinence de poursuivre les efforts de recherche relatifs à ces clientèles pour les années à venir. Souhaitons que notre bilan soit utile aux chercheurs qui contribueront à l'avancement des connaissances dans ce domaine.

Tableau 1 : Synthèse des études recensées

\begin{tabular}{|c|c|c|c|c|c|}
\hline Auteur(e)s & $\begin{array}{l}\text { Année } \\
\text { (type) }\end{array}$ & Région & Population & Méthode & Objectifs (continuum de l'intervention) \\
\hline Abdel Ghaly & $\begin{array}{c}2013 \\
(\mathrm{M})\end{array}$ & Québec & 8 femmes autochtones & Qualitative & $\begin{array}{l}\text { Explorer les représentations des } \\
\text { femmes autochtones à l'égard des } \\
\text { délais de placement maximaux } \\
\text { (Intervention) }\end{array}$ \\
\hline Belony & $\begin{array}{c}2007 \\
(\mathrm{M})\end{array}$ & Montréal & $\begin{array}{l}584 \text { jeunes de } 0 \text { à } 12 \text { ans pris en } \\
\text { charge par le Centre jeunesse de } \\
\text { Montréal } \\
\text { non immigrants ( } 58 \%) \text {, haïtiens } \\
\text { (18\%) et immigrants d'origine } \\
\text { autre qu'haïtienne }(76 \%)\end{array}$ & Quantitative & $\begin{array}{l}\text { Analyser la surreprésentation d'enfants } \\
\text { haïtiens parmi les enfants pris en } \\
\text { charge par la DPJ } \\
\text { Comparer les caractéristiques familiales } \\
\text { et le parcours de prise en charge des } \\
\text { trois groupes } \\
\text { (Intervention) }\end{array}$ \\
\hline Bergheul & $\begin{array}{c}2012 \\
(\mathrm{~A})\end{array}$ & $\begin{array}{l}\text { Montréal et } \\
\text { Algérie }\end{array}$ & $\begin{array}{l}656 \quad \text { jeunes contrevenants } \\
\text { québécois ( } 77 \% \text { garçons) de } 15 \text { à } \\
17 \text { ans } \\
124 \text { jeunes délinquants algériens } \\
\text { ( } 64 \% \text { garçons) de } 12 \text { à } 19 \text { ans }\end{array}$ & Quantitative & $\begin{array}{l}\text { Comparer deux études sur les conduites } \\
\text { marginales des adolescents au Québec } \\
\text { et en Algérie } \\
\text { (Intervention) }\end{array}$ \\
\hline $\begin{array}{l}\text { Bernard } \\
\text { McAll }\end{array}$ & $\begin{array}{c}2009 \\
(I)\end{array}$ & Montréal & $\begin{array}{l}\text { Tous les jeunes arrêtés à } \\
\text { Montréal en } 2001 \text { et dont le } \\
\text { dossier a été retenu en vertu de } \\
\text { la LSJPA } \\
175 \text { jeunes dont } 91 \text { jeunes } \\
\text { «noirs» }\end{array}$ & Quantitative & $\begin{array}{l}\text { Analyser la surreprésentation de jeunes } \\
\text { " noirs " montréalais dans le système } \\
\text { pénal juvénile et comparer les } \\
\text { trajectoires des jeunes "noirs " à celles } \\
\text { des jeunes « blancs" } \\
\text { (Intervention) }\end{array}$ \\
\hline $\begin{array}{l}\text { Bousquet St- } \\
\text { Laurent, } \\
\text { Dufour et } \\
\text { Lavergne }\end{array}$ & $\begin{array}{l}2014 \\
(I)\end{array}$ & $\mathrm{n} / \mathrm{a}$ & $\begin{array}{l}29 \text { pratiques d'intervention de la } \\
\text { maltraitance en contexte de } \\
\text { diversité culturelle recensées }\end{array}$ & Analyse doc. & $\begin{array}{l}\text { Recenser et critiquer les pratiques de } \\
\text { protection de l'enfance spécifiques aux } \\
\text { enfants issus de la diversité } \\
\text { ethnoculturelle et à leur famille } \\
\text { (Intervention) }\end{array}$ \\
\hline $\begin{array}{l}\text { Breton, } \\
\text { Dufour et } \\
\text { Lavergne }\end{array}$ & $\begin{array}{c}2012 \\
(\mathrm{~A})\end{array}$ & $\begin{array}{l}\text { Province de } \\
\text { Québec }\end{array}$ & $\begin{array}{l}1017 \text { enfants autochtones et } \\
15512 \text { non autochtones de } 0 \text { à } 17 \\
\text { ans et ayant fait l'objet d'un } \\
\text { signalement au moins une fois, } \\
\text { répartis dans } 9 \text { Centres jeunesse }\end{array}$ & Quantitative & $\begin{array}{l}\text { Évaluer la surreprésentation des } \\
\text { Autochtones en protection de l'enfance } \\
\text { Comparer le profil de ces enfants et de } \\
\text { leur famille en fonction d'un ensemble } \\
\text { de caractéristiques psychosociales } \\
\text { (Intervention) }\end{array}$ \\
\hline
\end{tabular}




\begin{tabular}{|c|c|c|c|c|}
\hline Brisebois & $\begin{array}{c}2010 \\
(\mathrm{M})\end{array}$ & Montréal & $\begin{array}{l}11 \text { mères et } 1 \text { oncle nés dans les } \\
\text { Antilles et dont le fils ou le neveu } \\
\text { est jeune contrevenant }\end{array}$ & Qualitative \\
\hline $\begin{array}{l}\text { Conseil du } \\
\text { statut de la } \\
\text { femme }\end{array}$ & $\begin{array}{c}2013 \\
\text { (I) }\end{array}$ & $\begin{array}{l}\text { Montréal et } \\
\text { autres }\end{array}$ & $\begin{array}{l}14 \text { femmes issues de diverses } \\
\text { communautés influencées par le } \\
\text { concept de l'honneur } \\
11 \text { personnes intervenant auprès } \\
\text { de femmes sujettes aux violences } \\
\text { basées sur l'honneur } \\
13 \text { experts sur le sujet }\end{array}$ & Qualitative \\
\hline $\begin{array}{l}\text { Couture et } \\
\text { Dufour }\end{array}$ & $\begin{array}{c}2013 \\
(\mathrm{~A})\end{array}$ & Montréal & $\begin{array}{l}24 \text { intervenants en protection de } \\
\text { l'enfance }\end{array}$ & Qualitative \\
\hline
\end{tabular}

\begin{tabular}{|c|c|c|c|}
\hline De Laco & $\begin{array}{c}2006 \\
(\mathrm{M})\end{array}$ & Montréal & $\begin{array}{l}50 \text { jeunes membres de gangs } \\
\text { détenus dans } 6 \text { unités à sécurité } \\
\text { maximale } \\
10 \text { Québécois francophones, } 10 \\
\text { Haïtiens, } 10 \text { Jamaïcains, } 10 \\
\text { Latinos-américains et } 10 \text { d'autres } \\
\text { origines }\end{array}$ \\
\hline $\begin{array}{l}\text { Desruisseaux } \\
\text { et al. }\end{array}$ & $\begin{array}{c}2002 \\
(\mathrm{~A})\end{array}$ & Montréal & $\begin{array}{l}122 \text { jeunes haïtiens répartis dans } \\
3 \text { écoles secondaires, âgés de } 14 \text { à } \\
19 \text { ans }\end{array}$ \\
\hline
\end{tabular}

Désy,

Battaglini et

Bastien

Hassan et

Lavergne
2007

(I)

Montréal

.

(1)

$\mathrm{n} / \mathrm{a}$

(C) l'enfance

$\mathrm{n} / \mathrm{a}$
315 intervenants en protection de

Qualitative

Quantitative

Analyse doc.

Tester le modèle de prédiction de la déviance selon lequel la privation relative collective serait associée à la méfiance raciale et à la fréquentation d'amis délinquants

(Prévention)

Identifier les facteurs permettant de qualifier et de quantifier l'intensité de l'intervention auprès des jeunes issus de l'immigration et de leur famille (Intervention)

Réfléchir sur les liens entre la maltraitance et la diversité culturelle, la surreprésentation des enfants de minorités visibles dans les services de protection de l'enfance et l'efficacité des interventions visant la maltraitance en contexte de diversité culturelle (Intervention)

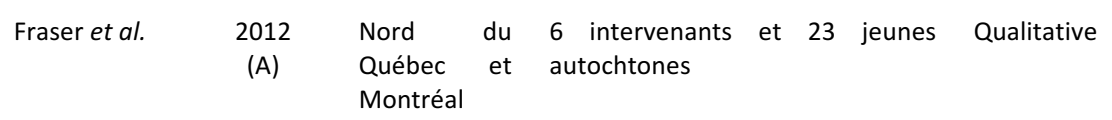

Documenter l'implantation d'un programme de réadaptation avec hébergement hors territoire pour jeunes Inuit

Identifier les obstacles et les éléments facilitateurs à la réintégration des jeunes dans la communauté (Réadaptation)

Documenter la perception des Autochtones relativement à leur situation de prise en charge (Intervention) 


\begin{tabular}{|c|c|c|c|c|c|}
\hline Garland & $\begin{array}{c}2009 \\
(\mathrm{M})\end{array}$ & Montréal & $\begin{array}{l}108 \text { jeunes évalués suite à un } \\
\text { signalement pour abus physique, } \\
\text { âgés de } 13 \text { à } 17 \text { ans } \\
51 \text { jeunes de minorités visibles }\end{array}$ & Quantitative & $\begin{array}{l}\text { Identifier les caractéristiques des } \\
\text { adolescents issus de minorités visibles } \\
\text { faisant l'objet d'un signalement pour } \\
\text { abus physique en comparaison aux } \\
\text { jeunes Caucasiens } \\
\text { (Intervention) }\end{array}$ \\
\hline Giroux & $\begin{array}{c}2012 \\
(\mathrm{M})\end{array}$ & Montréal & $\begin{array}{l}16 \text { intervenants en protection de } \\
\text { l'enfance }\end{array}$ & Quantitative & $\begin{array}{l}\text { Évaluer les effets et la mise en œuvre } \\
\text { du programme d'intervention "Vivre la } \\
\text { diversité » } \\
\text { (Intervention) }\end{array}$ \\
\hline $\begin{array}{l}\text { Goyette et } \\
\text { al. }\end{array}$ & $\begin{array}{l}2010 \text { et } \\
2012 \\
\text { (I) }\end{array}$ & $\begin{array}{l}\text { Abitibi- } \\
\text { Témiscaming } \\
\text { ue }\end{array}$ & $\begin{array}{l}31 \text { jeunes autochtones suivis ou } \\
\text { placés en Centre jeunesse, âgés } \\
\text { de } 16 \text { et } 17 \text { ans }\end{array}$ & Mixte & $\begin{array}{l}\text { Recenser les pratiques exemplaires } \\
\text { d'intervention de groupe destinées aux } \\
\text { jeunes en transition à la vie adulte } \\
\text { Évaluer l'implantation et les effets de } \\
\text { ces pratiques } \\
\text { (Réinsertion) }\end{array}$ \\
\hline $\begin{array}{l}\text { Goyette } \\
\text { al. }\end{array}$ & $\begin{array}{l}2009 \\
(1)\end{array}$ & $\begin{array}{l}\text { Abitibi- } \\
\text { Témiscaming } \\
\text { ue }\end{array}$ & $\begin{array}{l}4 \text { intervenants en Centre } \\
\text { jeunesse, } 17 \text { intervenants du } \\
\text { réseau de la santé et des services } \\
\text { sociaux ou de l'éducation et } \\
2 \text { membres d'un conseil de bande }\end{array}$ & Qualitative & $\begin{array}{l}\text { Documenter le processus d'insertion } \\
\text { sociale des jeunes algonquins qui } \\
\text { doivent quitter les centres jeunesse à } \\
\text { l'approche de l'âge adulte } \\
\text { Mettre en œuvre et évaluer des } \\
\text { stratégies d'intervention de groupe } \\
\text { visant la réduction de la victimisation et } \\
\text { la prévention du crime } \\
\text { (Réinsertion) }\end{array}$ \\
\hline $\begin{array}{l}\text { Goyette, } \\
\text { Pontbriand } \\
\text { et Turcotte }\end{array}$ & $\begin{array}{c}2011 \\
(\text { (I) }\end{array}$ & $\begin{array}{l}\text { Abitibi- } \\
\text { Témiscaming } \\
\text { ue }\end{array}$ & $\begin{array}{l}17 \text { intervenants d'un Centre } \\
\text { jeunesse et travaillant auprès de } \\
\text { familles autochtones }\end{array}$ & Qualitative & $\begin{array}{l}\text { Évaluer l'impact des modifications } \\
\text { apportées à la LPJ en } 2007 \text { sur } \\
\text { l'intervention d'équipes intervenant } \\
\text { auprès de la clientèle autochtone } \\
\text { (Intervention) }\end{array}$ \\
\hline Grenier et al. & $\begin{array}{c}2013 \\
(A)\end{array}$ & $\begin{array}{l}\text { Abitibi- } \\
\text { Témiscaming } \\
\text { ue }\end{array}$ & $\begin{array}{l}2 \text { intervenants et } 9 \text { jeunes } \\
\text { autochtones de } 16 \text { à } 21 \text { ans }\end{array}$ & Qualitative & $\begin{array}{l}\text { Évaluer l'implantation et les résultats du } \\
\text { programme } D^{\prime} h i e r \text { à demain visant à } \\
\text { soutenir le passage à la vie adulte chez } \\
\text { de jeunes autochtones ayant été placés } \\
\text { en Centre jeunesse } \\
\text { (Réinsertion) }\end{array}$ \\
\hline Grobe & $\begin{array}{l}2000 \\
(\mathrm{M})\end{array}$ & $\begin{array}{l}\text { Péninsule } \\
\text { Québec- } \\
\text { Labrador }\end{array}$ & $\begin{array}{l}84 \text { adolescents de } 11 \text { àt } 17 \text { ans } \\
61 \text { Autochtones }\end{array}$ & Quantitative & $\begin{array}{l}\text { Évaluer l'association entre les relations } \\
\text { d'attachement insécurisé et des } \\
\text { problèmes de comportement chez les } \\
\text { adolescents de Premières Nations } \\
\text { provenant d'un milieu géographique } \\
\text { éloigné } \\
\text { (Intervention) }\end{array}$ \\
\hline $\begin{array}{l}\text { Hassan et } \\
\text { Rousseau }\end{array}$ & $\begin{array}{l}2007 \\
(A)\end{array}$ & Montréal & $\begin{array}{l}5 \text { intervenants en protection de } \\
\text { l'enfance }\end{array}$ & Qualitative & $\begin{array}{l}\text { Explorer les perceptions, le vécu, les } \\
\text { réussites et les défis rencontrés par des } \\
\text { intervenants en protection de l'enfance } \\
\text { en contexte de diversité culturelle } \\
\text { Documenter les facteurs facilitant } \\
\text { l'intervention en contexte de diversité } \\
\text { culturelle } \\
\text { (Intervention) }\end{array}$ \\
\hline $\begin{array}{l}\text { Hassan et } \\
\text { Rousseau }\end{array}$ & $\begin{array}{l}2009 \\
(A)\end{array}$ & Montréal & $\begin{array}{l}12 \text { dyades parent-adolescent ainsi } \\
\text { que } 8 \text { parents et } 8 \text { adolescents } \\
\text { non apparentés } \\
50 \% \text { originaires du Maghreb, } \\
50 \% \text { originaires d'Amérique } \\
\text { Latine }\end{array}$ & Qualitative & $\begin{array}{l}\text { Documenter les valeurs relatives à la } \\
\text { discipline physique chez des parents et } \\
\text { des adolescents immigrants } \\
\text { (Prévention) }\end{array}$ \\
\hline
\end{tabular}




\begin{tabular}{|c|c|c|c|c|c|}
\hline Hélie et al. & $\begin{array}{c}2013 \\
(A)\end{array}$ & $\begin{array}{l}\text { Province } \\
\text { Québec }\end{array}$ & $\begin{array}{l}25897 \text { jeunes de } 0 \text { à } 17 \text { ans dont } \\
\text { la prise en charge par les services } \\
\text { de protection s'est terminée } \\
\text { entre } 2005 \text { et } 2006 \\
6 \% \text { de jeunes autochtones }\end{array}$ & Quantitative & $\begin{array}{l}\text { Identifier les facteurs de risque de } \\
\text { première récurrence en protection de } \\
\text { l'enfance durant les cinq ans suivant la } \\
\text { fermeture de la première prise en } \\
\text { charge } \\
\text { (Intervention) }\end{array}$ \\
\hline Hélie et al. & $\begin{array}{c}2011 \\
\text { (I) }\end{array}$ & $\begin{array}{l}\text { Province } \\
\text { Québec }\end{array}$ & $\begin{array}{l}9623 \text { enfants protégés avant les } \\
\text { changements à la LPJ } \\
8991 \text { enfants protégés après les } \\
\text { changements à la LPJ } \\
7 \% \text { d'enfants autochtones }\end{array}$ & Quantitative & $\begin{array}{l}\text { Établir dans quelle mesure les nouvelles } \\
\text { dispositions de la LPJ contribuent à } \\
\text { répondre aux besoins des enfants dont } \\
\text { la sécurité ou le développement est } \\
\text { compromis en assurant la stabilité de } \\
\text { leurs conditions de vie } \\
\text { (Intervention) }\end{array}$ \\
\hline Hélie et al. & $\begin{array}{c}2012 \\
(1)\end{array}$ & $\begin{array}{l}\text { Province } \\
\text { Québec }\end{array}$ & $\begin{array}{l}3079 \text { enfants ayant fait l'objet } \\
\text { d'une évaluation suite à un } \\
\text { signalement dans un Centre } \\
\text { jeunesse du Québec en } 2008 \\
5 \% \text { d'enfants autochtones }\end{array}$ & Quantitative & $\begin{array}{l}\text { Produire des estimations fiables des } \\
\text { caractéristiques et de l'ampleur des } \\
\text { signalements au Québec } \\
\text { (Intervention) }\end{array}$ \\
\hline Jimenez et al. & $\begin{array}{c}2003 \\
(1)\end{array}$ & Montréal & $\begin{array}{l}191 \text { enfants de } 6 \text { à } 12 \text { ans } \\
96 \text { enfants d'immigration récente }\end{array}$ & Quantitative & $\begin{array}{l}\text { Documenter l'impact de l'exposition à } \\
\text { la violence conjugale chez les enfants } \\
\text { issus d'une famille d'immigration } \\
\text { récente } \\
\text { (Prévention) }\end{array}$ \\
\hline Labonté & $\begin{array}{c}2010 \\
(\mathrm{M})\end{array}$ & Québec & $\begin{array}{l}11 \text { parents immigrants réfugiés } \\
5 \text { de ces parents recevaient des } \\
\text { services en protection de } \\
\text { l'enfance }\end{array}$ & Qualitative & $\begin{array}{l}\text { Étudier les représentations des parents } \\
\text { réfugiés par rapport aux services de } \\
\text { protection de l'enfance } \\
\text { (Intervention) }\end{array}$ \\
\hline $\begin{array}{l}\text { Lavergne, } \\
\text { Dufour et } \\
\text { Couture }\end{array}$ & $\begin{array}{c}2013 \\
(A)\end{array}$ & Montréal & $\begin{array}{l}22 \text { intervenants en protection de } \\
\text { l'enfance }\end{array}$ & Qualitative & $\begin{array}{l}\text { Recueillir leur point de vue } \\
\text { d'intervenants sur les causes et les } \\
\text { pistes de solution à la disproportion des } \\
\text { jeunes issus de l'immigration } \\
\text { (Intervention) }\end{array}$ \\
\hline $\begin{array}{l}\text { Lavergne et } \\
\text { al. }\end{array}$ & $\begin{array}{l}2009 \\
(A)\end{array}$ & Montréal & $\begin{array}{l}3918 \text { enfants dont le signalement } \\
\text { a été retenu entre juillet } 2007 \text { et } \\
\text { juillet } 2008 \\
12 \% \text { d'enfants «noirs » } \\
13 \% \text { enfants issus d'autres } \\
\text { minorités ethniques }\end{array}$ & Quantitative & $\begin{array}{l}\text { Comparer la situation des enfants non } \\
\text { issus des minorités visibles, les enfants } \\
\text { noirs et les enfants issus d'autres } \\
\text { minorités visibles qui font l'objet d'une } \\
\text { évaluation par les services de } \\
\text { protection montréalais } \\
\text { (Intervention) }\end{array}$ \\
\hline Leblanc & $\begin{array}{c}2003 \\
(\mathrm{M})\end{array}$ & Non précisée & $\begin{array}{l}4 \text { travailleurs sociaux en pro- } \\
\text { tection de l'enfance et d'origine } \\
\text { autochtone }\end{array}$ & Qualitative & $\begin{array}{l}\text { Explorer les implications politiques qui } \\
\text { influencent le sens que les travailleurs } \\
\text { sociaux autochtones attribuent à leur } \\
\text { travail communautaire } \\
\text { (Intervention) }\end{array}$ \\
\hline $\begin{array}{l}\text { McGinn } \\
\text { Damase }\end{array}$ & $\begin{array}{l}2014 \\
(I)\end{array}$ & $\mathrm{n} / \mathrm{a}$ & $\mathrm{n} / \mathrm{a}$ & $\begin{array}{l}\text { Revue } \\
\text { systématique } \\
\text { d'écrits }\end{array}$ & $\begin{array}{l}\text { Identifier les interventions préventives } \\
\text { ou thérapeutiques efficaces en milieu } \\
\text { autochtone en situation de négligence } \\
\text { Identifier les facteurs favorisant } \\
\text { l'implantation de ces mesures } \\
\text { (Intervention) }\end{array}$ \\
\hline Mitchell & $\begin{array}{c}2011 \\
(\mathrm{M})\end{array}$ & $\begin{array}{l}\text { Nord } \\
\text { Québec }\end{array}$ & $\begin{array}{l}27 \text { adultes membres de la com- } \\
\text { munauté autochtone Naskapi }\end{array}$ & Qualitative & $\begin{array}{l}\text { Documenter la perception des mesures } \\
\text { de retrait et de placement à l'extérieur } \\
\text { de la communauté auprès de membres } \\
\text { de la communauté Naskapi } \\
\text { (Intervention) }\end{array}$ \\
\hline
\end{tabular}




\begin{tabular}{|c|c|c|c|c|c|c|}
\hline $\begin{array}{l}\text { Morin } \\
\text { Lafortune }\end{array}$ & et & $\begin{array}{c}2008 \\
(A)\end{array}$ & $\begin{array}{l}\text { Nord du } \\
\text { Québec }\end{array}$ & $\begin{array}{l}6 \text { infirmières, } 3 \text { travailleurs com- } \\
\text { munautaires, } 6 \text { intervenants non } \\
\text { diplômés } \\
8 \text { de ces adultes sont d'origine } \\
\text { inuite }\end{array}$ & Qualitative & $\begin{array}{l}\text { Décrire la perception d'intervenants } \\
\text { relativement au contexte de } \\
\text { victimisation sexuelle en milieu inuit et } \\
\text { aux relations entre intervenants } \\
\text { allochtones et Inuits } \\
\text { (Intervention) }\end{array}$ \\
\hline Muckle & & $\begin{array}{c}2011 \\
(T)\end{array}$ & Mauricie & $\begin{array}{l}227 \text { jeunes autochtones de } 14 \text { à } \\
19 \text { ans }\end{array}$ & Quantitative & $\begin{array}{l}\text { Mieux comprendre les facteurs qui } \\
\text { modèrent l'effet du trauma infligé par } \\
\text { l'agression sexuelle chez les } \\
\text { Autochtones } \\
\text { (Intervention) }\end{array}$ \\
\hline $\begin{array}{l}\text { Perreault } \\
\text { Bibeau }\end{array}$ & et & $\begin{array}{c}2003 \\
(L)\end{array}$ & Montréal & $\begin{array}{l}55 \quad \text { jeunes Afro-Antillais } \\
\text { marginalisés, âgés de } 13 \text { à } 22 \text { ans }\end{array}$ & Qualitative & $\begin{array}{l}\text { Décrire le point de vue des jeunes } \\
\text { relativement au phénomène de la } \\
\text { marginalité } \\
\text { Reconnaître les conditions menant à } \\
\text { l'entrée dans la gang, au maintien de } \\
\text { l'appartenance et les conditions } \\
\text { favorisant ou non l'abandon du groupe } \\
\text { (Réinsertion) }\end{array}$ \\
\hline Robitaille & & $\begin{array}{c}2011 \\
(\mathrm{M})\end{array}$ & Non précisée & $\begin{array}{l}5 \text { intervenants en Centre jeunesse } \\
\text { desservant une clientèle } \\
\text { autochtone }\end{array}$ & Qualitative & $\begin{array}{l}\text { Examiner la manière dont la culture } \\
\text { autochtone est prise en compte dans } \\
\text { les processus décisionnels cliniques } \\
\text { (Intervention) }\end{array}$ \\
\hline Sigouin & & $\begin{array}{c}2006 \\
(\mathrm{M})\end{array}$ & $\mathrm{n} / \mathrm{a}$ & $n / a$ & Analyse doc. & $\begin{array}{l}\text { Explorer les mécanismes juridiques qui } \\
\text { permettent de mieux protéger les } \\
\text { enfants autochtones } \\
\text { (Intervention) }\end{array}$ \\
\hline $\begin{array}{l}\text { Sinha } \\
\text { Kozlowski }\end{array}$ & et & $\begin{array}{l}2013 \\
(\mathrm{~A})\end{array}$ & Canada & $n / a$ & Analyse doc. & $\begin{array}{l}\text { Décrire la structure des services de } \\
\text { protection de l'enfance spécifiques aux } \\
\text { Autochtones au Canada } \\
\text { (Intervention) }\end{array}$ \\
\hline $\begin{array}{l}\text { Tichit } \\
\text { Jaccoud }\end{array}$ & et & $\begin{array}{c}2009 \\
(I)\end{array}$ & Montréal & $\begin{array}{l}36 \text { adolescents, } 25 \text { jeunes adultes } \\
\text { et } 18 \text { parents haïtiens, africains } \\
\text { ou caribéens }\end{array}$ & Qualitative & $\begin{array}{l}\text { Rapporter les perceptions de membres } \\
\text { des communautés noires à Montréal } \\
\text { relativement à la violence } \\
\text { (Prévention) }\end{array}$ \\
\hline $\begin{array}{l}\text { Tourigny } \\
\text { al. }\end{array}$ & et & $\begin{array}{c}2007 \\
(\mathrm{~A})\end{array}$ & $\begin{array}{l}\text { Abitibi- } \\
\text { Témiscaming } \\
\text { ue et Côte- } \\
\text { Nord }\end{array}$ & $\begin{array}{l}215 \text { jumelages d'enfants ayant } \\
\text { des caractéristiques comparables } \\
\text { Un enfant autochtone et un } \\
\text { enfant non autochtone pour } \\
\text { chaque paire d'enfants }\end{array}$ & Quantitative & $\begin{array}{l}\text { Examiner les différences et les } \\
\text { similarités pouvant exister entre les } \\
\text { dossiers des enfants autochtones et } \\
\text { non autochtones signalés aux services } \\
\text { de protection de l'enfance } \\
\text { (Intervention) }\end{array}$ \\
\hline Tremblay & & $\begin{array}{l}2008 \\
(I)\end{array}$ & Montréal & $\begin{array}{l}16 \text { jeunes d'origine Latino ayant } \\
\text { fréquenté des gangs de rue et } \\
\text { âgés de } 14 \text { à } 22 \text { ans }\end{array}$ & Qualitative & $\begin{array}{l}\text { Décrire l'expérience de jeunes latinos } \\
\text { ayant fréquenté de gangs de rue à } \\
\text { Montréal ainsi que le contexte familial } \\
\text { et social les entourant } \\
\text { (Prévention) }\end{array}$ \\
\hline
\end{tabular}

Abréviations : A, article; C, chapitre de livre; doc., documntaire; I; institutionnel; L, livre; M, mémoire; T, thèse.

\section{Références bibliographiques}

Abdel Ghaly, M. (2013). Perspectives de femmes autochtones en milieu urbain sur les délais de placement maximaux: étude exploratoire (Mémoire de maîtrise inédit). Université Laval, Québec.

Belony, E. (2007). La prise en charge des enfants de l'immigration haitienne par la Direction de la protection de la jeunesse : une analyse comparative (Mémoire de maîtrise inédit). Université de Montréal. 
Bergheul, S. (2012). La délinquance des adolescentes: étude comparative entre une population canadienne et algérienne. Neuropsychiatrie de l'enfance et de l'adolescence, 60(6), 446-453.

Bernard, L. et McAll, C. (2009). Jeunes, police et système de justice. La surreprésentation des jeunes noirs montréalais. Montréal, Québec: Centre de recherche de Montréal sur les inégalités sociales, les discriminations et les pratiques alternatives de citoyenneté.

Bousquet St-Laurent, T., Dufour, S. et Lavergne, C. (2014). Bilan critique des pratiques associées à la diversité ethnoculturelle en protection de la jeunesse. Montréal : Centre jeunesse de Montréal-Institut universitaire.

Breton, A., Dufour, S. et Lavergne, C. (2012). Les enfants autochtones en protection de la jeunesse au Québec : leur réalité comparée à celle des autres enfants. Criminologie, 45(2), 157-185.

Brisebois, R.-A. (2007). Parcours de parents immigrants dont le fils a fait l'expérience d'un gang de rue et d'une institutionnalisation: un vécu familial bouleversé (Mémoire de maîtrise inédit). Université de Montréal.

Chamberland, C. (1998). L'approche milieu dans les Centres jeunesse de Montréal : vers une nouvelle culture de l'intervention. Défi jeunesse, 4(3).

Chand, A. et Thoburn, J. (2005). Child and family support services with minority ethnic families: What can we learn from research? Child and Family Social Work, 10(2), 169-178.

Centre intégré universitaire de santé et de services sociaux (CIUSS) du Centre-Sud-de-I'île-de-Montréal (2015). Programmation scientifique du Centre de recherche Jeunes en difficulté. En ligne à : http://www.centrejeunessedemontreal.qc.ca/recherche/Prog_Scientifique.php

Conseil du statut de la femme (2013). Les crimes d'honneur : de l'indignation à l'action. Québec : Conseil du statut de la femme.

Couture, D. et Dufour, S. (2013). Collaboration des minorités visibles avec les services de protection: soutien informel et stratégies d'intervention. Revue québécoise de psychologie, 34(2), 147-170.

De Laco, G. A. (2006). Juvenile street gang members and ethnic identity in Montréal, Canada (Mémoire de maîtrise inédit). Université McGill, Montréal.

Desruisseaux, J-C., St-Pierre, L., Tougas, F. et de la Sablonnière, R. (2002). Jeunes haïtiens de Montréal et déviance : frustration, méfiance et mauvaises fréquentations. Revue Québécoise de Psychologie, 23(3), 43-55.

Désy, M., Battaglini, A. et Bastien, R. (2007). Mesure de l'intensité nécessaire à l'intervention auprès des jeunes immigrants et leur famille: Centre jeunesse de Montréal et Centre de la jeunesse et de la famille Batshaw. Montréal : Agence de la santé et des services sociaux de Montréal.

Dufour, S., Hassan, G. et Lavergne, C. (2012). Mauvais traitements et diversité culturelle: bilan des connaissances et implications pour la pratique. Dans M-H Gagné, S. Drapeau et M-C Saint-Jacques (dir.), Les enfants maltraités : de l'affliction à l'espoir : pistes de compréhension et d'action (p. 143-171). Québec : Presses de I'Université Laval.

Feiring, C. et Zielinski, M. (2011). Looking back and looking forward : A review and reflection on research articles published in child maltreatment from 1996 to 2010. Child Maltreatment, 16(1), 3-8.

Fraser, S. L, Vachon, M, Arauz, M. J., Rousseau, C. et Kirmayer, L. J. (2012). Inuit youth transitioning out of residential care: Obstacles to re-integration and challenges to wellness. First Peoples Child and Family Review, 7(1), 52-75.

Gagnon-Dion, M.-H. (2014). Entre déracinement et émancipation: I'expérience des jeunes autochtones pris en charge par la protection de la jeunesse (Mémoire de maîtrise inédit). Université de Montréal, Montréal.

Garland, L. (2009). Does culture matter? The characteristics of adolescent physical abuse cases investigated by Montreal's English youth protection services (Mémoire de maîtrise inédit). Université McGill, Montréal.

Giroux, J. (2012). Rapport d'intervention du programme Vivre la diversité ! (Ateliers de formation sur les relations interculturelles en contexte d'intervention de crise) (Mémoire de maîtrise inédit). Université de Montréal, 
Montréal. En ligne à :

https://papyrus.bib.umontreal.ca/xmlui/bitstream/handle/1866/9772/2012_Giroux_Joannie.pdf

Goyette, M., Grenier, S., Mann-Feder, V., Turcotte, D., Grenier, S., Turcotte, M-È, Pontbriand, A et Bamba, E-H (2010). Parcours d'entrée dans la vie adulte et stratégies d'autonomisation: une lecture dynamique des trajectoires de jeunes autochtones suivis ou placés en protection de la jeunesse. Montréal : École nationale d'administration publique (ENAP) et Chaire de recherche du Canada sur l'évaluation des actions publiques à l'égard des jeunes et des populations vulnérables.

Goyette, M., Grenier, S., Pontbriand, A., Turcotte, M-È, Royer, M-N et Corneau, M. (2009). Enjeux relatifs au passage à la vie adulte de jeunes autochtones : vers une meilleure compréhension de la particularité du contexte de communauté algonquines de l'Abitibi. Montréal : École nationale d'administration publique (ENAP).

Goyette, M., Mann-Feder, V., Turcotte, D., Grenier, S., Turcotte, M. - E., Plagès, M., Pontbriand, A. et Corneau, M. (2012). Évaluation de l'implantation et des effets d'interventions de groupe visant à soutenir le passage à la vie adulte de jeunes issus des centres jeunesse et de jeunes autochtones : rapport final d'évaluation des groupes 1 à 6. Montréal, Québec: CREVAJ, École nationale d'administration publique.

Goyette, M., Pontbriand, A. et Turcotte, M.-È. (2011). Les services offerts aux communautés autochtones par un Centre jeunesse. Dans D. Turcotte et collab. (dir.), Évaluation des impacts de la nouvelle Loi sur la protection de la jeunesse - programme Actions concertées (p. 271-304). Rapport présenté au CNPC.

Grenier, S., Goyette, M., Turcotte, D., Mann-Feder, V. et Turcotte, M-È (2013). L'intervention de groupe pour soutenir le passage à la vie adulte des jeunes autochtones issus des centres jeunesse et de deux communautés. First Peoples Child and Family Review, 7(2), 148-159.

Grobe, P. (2000). Attachment and delinquency among First Nations adolescents from a remote geographic location (Mémoire de maîtrise inédit). Université McGill, Montréal.

Hassan, G. et Rousseau, C. (2007). La protection des enfants : enjeux de l'intervention en contexte interculturel. Bulletin de l'Association pour la recherche interculturelle, 45, 37-50.

Hassan, G. et Rousseau, C. (2009). Quand la divergence devient exclusion : perceptions des châtiments corporels par les parents et les adolescents immigrants. Revue Transculturelle, 10(3), 292-304.

Hélie, S. et Laurier, C. (2012). Récurrence de la violence, subie ou agie, après la fermeture d'un premier signalement avec prise en charge en protection de la jeunesse : ampleur du risque et facteurs associés. Rapport déposé à la Table des directeurs des services professionnels (DSP) de l'Association des centres jeunesse du Québec (ACJQ).

Hélie, S., Laurier, C., Pineau-Villeneuve, C. et Royer, M-N. (2013). A developmental approach to the risk of a first recurrence in child protective services. Child Abuse and Neglect, 37(12), 1132-1141.

Hélie, S., Turcotte, D., Royer, M-N et Lamonde, G. (2011). L'impact de la nouvelle LPJ sur la stabilité des enfants placés. Dans D. Turcotte et al., Évaluation des impacts de la nouvelle Loi sur la protection de la la jeunesse au Québec, rapport final déposé au FQRSC, programme Actions concertées.

Hélie, S., Turcotte, D., Trocmé, N. et Tourigny, M. (2012). Étude d'incidence québécoise sur les situations évaluées en protection de la jeunesse en 2008 (ÉlQ-2008), rapport final. Rapport de recherche CJM-IU, Montréal.

Jimenez, V., Saucier, J-F., Marleau, J. D., Murphy, C., Ciampi, A., Côté, B. et Tong, G. (2003). Impact du fait d'être témoin de violence conjugale sur la santé mentale d'enfants âgés de 6 à 12 ans de familles d'immigration récente et québécoises. Rapport présenté au Conseil québécois de la recherche sociale et à la Régie régionale de la santé et des services sociaux de Montréal-Centre.

Kroes, G. (2008). Les jeunes Autochtones du Canada : nouveaux enjeux, priorités de recherche et incidences politiques. Ottawa : Gouvernement du Canada. (Projet de recherche sur les politiques) 
Labonté, M-H (2010). La protection de la jeunesse vue par des parents réfugiés: la famille au cœur de la protection de la jeunesse (Mémoire de maîtrise inédit). Université Laval, Québec.

Latimer, J. et Foss, L. C. (2004). Profil instantané d'une journée des jeunes Autochtones sous garde au Canada. Ottawa : Division de la recherche et de la statistique, Justice Canada. En ligne à : http//:www.canada.justice.gc.ca/eng/pi/rs/reprap/2004/yj2-jj2/index.html

Lavergne, C., Dufour, S. et Couture, D. (2013). Point de vue des intervenants sur la disproportion des enfants issus de groupes racialisés dans le système de protection de la jeunesse : causes et pistes de solution. Alterstice, $4(1), 17-30$.

Lavergne, C., Dufour, S., Sarmiento, J. et Descôteaux, M-È. (2009). La réponse du système de protection de la jeunesse montréalaise aux enfants issus des minorités visibles. Intervention, 131, 233-241.

Leblanc, D. (2003). Working in a post-colonial system: Whose voices are being silenced and heard in the narratives of Native child welfare workers? (Mémoire de maîtrise inédit). Université McGill, Montréal.

McGinn, C. A. et Damasse, J. (2014). Efficacité des interventions en matière de négligence auprès des enfants, des familles et des communautés autochtones. Québec: Institut national d'excellence en santé et en services sociaux.

Ministère de la Santé et des Services Sociaux (MSSS) (2005). Le développement de la mission universitaire dans les établissements des services sociaux. Québec : Gouvernement du Québec.

Mitchell, J. E. (2011). Creating an infrastructure of 'good practice' in child welfare in the community of Kawawachikamach (Mémoire de maîtrise inédit). Université McGill, Montréal.

Morin, E. et Lafortune, D. (2008). L'agression sexuelle à l'égard des mineurs en territoires nordiques: perceptions des intervenants. Revue canadienne de santé mentale communautaire, 27(1), 93-110.

Morneau, N. (1999). Réflexions sur l'intervention en maltraitance auprès des groupes culturels minoritaires. Revue canadienne de service social, 16(2), 219-231.

Muckle, F. (2011). La résilience psychologique et les facteurs de protections en contexte d'agression sexuelle auprès d'adolescents autochtones et caucasiens : une étude exploratoire (Thèse de doctorat inédite). Université du Québec à Chicoutimi, Chicoutimi.

Perreault, M. et Bibeau, G. (2003). La gang : une chimère à apprivoiser. Marginalité et transnationalité chez les jeunes Québécois d'origine afro-antillaise. Montréal, Québec : Boréal.

Petersen, N., Joseph, J. et Feit, M. (2015). New directions on child abuse and neglect research. Washington, Institute of Medecine National Council Research.

Robitaille, M. (2011). Les situations de compromission: regards professionnels sur les interventions auprès des Premières Nations (Mémoire de maîtrise inédit). Université d'Ottawa, Ottawa.

Saunders, B., Berliner, L. et Hanson, R. (2004). Child physical and sexual abuse: Guidelines for treatment. Charleston, SC: National Crime Victims Research and Treatment Center.

Sigouin, É. (2006). Les mécanismes de protection de la jeunesse autochtone au regard de la théorie libérale de Will Kymlicka (Mémoire de maîtrise). Université de Montréal, Montréal. https://papyrus.bib.umontreal.ca/xmlui/handle/1866/2411

Sinha, V. et Kozlowski, A. (2013). The structure of Aboriginal child welfare in Canada. The International Indigenous Policy Journal, 4(2), 1-21.

Statistique Canada (2010). Projections de la diversité de la population canadienne, 2006 à 2031. Récupéré de http://www.statcan.gc.ca/pub/91-551-x/91-551-x2010001-fra.pdf

Tichit, L. et Jaccoud, M. (2009). Black communities speak out about violence. Montréal : Réseau de communication pour la prévention des actes criminels (RECOPAC). 
Tourigny, M., Domond, P., Trocmé, N., Sioui, B. et Baril, K. (2007). Les mauvais traitements envers les enfants autochtones signalés à la Protection de la jeunesse du Québec: comparaison interculturelle. First Peoples Child and Family Review, 3(3), 84-102.

Tremblay, M. (2008). À la découverte des jeunes latinos qui fréquentent les gangs de rue à Montréal (Mémoire de maîtrise inédit). Université de Montréal, Montréal.

Tremblay, D., Moisan, S., Laquerre, C. et Faugeras, F. (2002). Les enjeux et les défis de l'intervention en Centre jeunesse. Module pédagogique. Québec : Centre jeunesse de Québec-institut universitaire.

Wells, K, Miranda, J., Bruce, M., Alegria, M. et Wallerstein, N. (2004). Bridging community intervention and mental health services research. American Journal of Psychiatry, 161(6), 955-963. 